\title{
UN RETRATO EN MEDALLA DEL CARDENAL PORTOCARRERO, POR GABINO VELÁZQUEZ
}

Cuando en el año 1831 reanudó por fin la Real Academia de Bellas Artes de San Fernando la celebración de los concursos generales estipulada en los estatutos, que no se convocaban desde su interrupción forzosa en 1808, el correspondiente a grabado de medallas concluyó con la concesión del primer premio (la consabida medalla de oro de una onza) a un joven de 28 años, de quien se dice ser madrileño, llamado Gabino Velázquez. Para el lector del acta de la competición ${ }^{1}$ informado del desarrollo posterior de esa disciplina artística en nuestro país, lo que antes llama la atención en ella es el muy diferente destino profesional que esperaba a tan prometedor estudiante respecto del que conocemos del principal de sus adversarios, Bartolomé Coromina ${ }^{2}$. Muy al contrario que éste ${ }^{3}$, Gabino Velázquez desaparece a partir de la fecha del concurso de la documentación artística conocida, incluyéndose en ella las mismas medallas, de las cuales ninguna firmada por él se halla recogida en la bibliografía sobre el tema. Esto puede deberse a ignorancia nuestra de un terreno insuficientemente explorado y que opone tantas trabas a su estudio; ignorancia, por otro lado, que no puede ser tan aguda que permita concebir que una trayectoria importante en su valor creativo pudiera haber sido pasada por alto. Además, el otro dato disponible hoy sobre su carrera de grabador ofrece un indicio seguro de la dirección en que debe buscarse el desenvolvimiento de aquélla: una Real Orden de 25 de abril de 1828 nombró a Gabino Velázquez primer alumno de segunda clase (de la talla) en el Departamento de Grabado de la ceca madrileña ${ }^{4}$. Así pues, las labores técnicas propias de un establecimiento de esta índole debieron absorber, como en tantos otros casos, su incipiente talento y el grueso, si no el total, de su actividad. Con ello quedó en nada, desde el punto de vista puramente artístico, la promesa que brillaba en los estudios académicos.

Entonces, si casi todo es oscuridad, ¿por qué ocuparse de él? El propósito de estas líneas es precisamente exponer dos razones. En primer lugar, su propia personalidad, puesto que el nombre de Gabino Velázquez corresponde, abreviado como era hábito en la familia, a Gabino González Velázquez, sexto hijo del pintor Zacarías (1763-1834) y de su esposa Juana Fernández y Ginés (1772-1809), nacido en 1803 residiendo sus padres en Aranjuez. Era, por consiguiente, miembro de una estirpe de artistas de importancia fundamental en el panorama español de la época, como se reconoce ya sin reparos. Gracias al magnífico trabajo de Bertha Núñez

${ }^{1}$ Distribución de los premios concedidos por el Rey Nuestro Señor a los discípulos de las Tres Nobles Artes, hecha por la Real Academia de San Fernando en la junta pública de 27 de marzo de 1832, Madrid, 1832, pp. 134 y 143 . El asunto propuesto a los concursantes, aprobado en junta ordinaria de 19 de diciembre de 1830, fue el siguiente: «La Real Academia de San Fernando representada por una matrona corona los atributos de las nobles artes. En rededor de la medalla se leerá 'Non coronabitur nisi legitime certaverit'. Lema adoptado por la Real Academia para manifestar que solo dispensa los premios á los que por su mérito se hacen dignos de ello [...] finalizado el término de seis meses, se señalaron los dias 26, 27 y 28 de Setiembre para la convocacion de las tres Juntas generales en que se debian hacer las pruebas de repente, votar y adjudicar los premios á los opositores mas benemeritos. Los asuntos que salieron en suerte para dichas pruebas segun sus clases son los siguientes [para el grabado de medallas:] Sansón luchando con el leon».

${ }^{2}$ Concurrieron al certamen cinco estudiantes: Lorenzo Barrio, Gabino Velázquez, Juan Plañiol, Francisco [González de] Miranda (véase Distribución, cit., p. 42) y Bartolomé Coromina. De ellos, dos votos recibieron respectivamente Coromina y Plañiol, frente a los tres de Velázquez. «Considerando la Academia cuanto convenia fomentar esta arte para el mejor servicio de las casas de moneda de S. M., acordó dar un premio estraordinario» (Distribución, cit., p. 143), que se decidió, entre los empatados y tras nuevo examen, por el barcelonés Coromina.

${ }^{3} \mathrm{El}$ cual fue hecho en 1844 académico de mérito; véase el Catálogo de los individuos de la Real Academia de Nobles Artes de S. Fernando en 31 de Agosto de 1845, Madrid, 1845, p. 44.

${ }^{4}$ Herrera, Adolfo, El Duro, Madrid, 1914, p. 499, información que el autor extrajo de documentos del archivo del Ministerio de Hacienda. « $4^{\circ}$. Los discípulos de la segunda clase (Artículo $7^{\circ}$ del Reglamento) será su principal destino de talladores, y se les instruirá para el desempeño de las obras de este Cargo, en sacar, y reparar punzones, y principalmente en la multiplicación de troqueles, el mecanismo del volante, y demás Instrumentos que es el principal objeto a que se les destina, uniendo como es preciso al menos una mediana habilidad en el Gravado»; cit. de la Instrucción ya mencionada. 
Vernis ${ }^{5}$, que dio a la luz numerosos documentos del archivo familiar, disponemos de un esquema suficiente de su biografía. Éste confirma el inicio, en 1834 y «estando al servicio de la Real Casa de Moneda», de una larga carrera administrativa que ocupó el resto de su vida, acabada el 21 de octubre de $1866^{6}$.

La segunda de mis razones viene a dar valor a la primera y a disipar en parte, aunque sea mínima, aquella tiniebla: la localización de una obra suya, firmada y fechada, la cual justificará con su presencia metálica la inclusión de su autor en el catálogo de medallistas españoles del siglo XIX cuya formación habrá de emprenderse algún día. Es cierto, sin embargo, que no lo hará aún con pleno derecho, porque la mencionada obra (Fig. 1) no es propiamente una medalla, sino una prueba de anverso sobre estaño, impresa «cuando el troquel está sin templar» ${ }^{7}$. Tiene $58 \mathrm{~mm}$. de diámetro, y la leyenda dice: LUDOV[ICUS] - PORTOCARRERO . $\mathrm{S}[\mathrm{ANCTAE}] \cdot \mathrm{R}[\mathrm{OMANAE}] \cdot \mathrm{E}[\mathrm{CCLESIAE}] \cdot$ CARDINALIS; bajo el busto, G. VELAZQUEZ · 1826. Su fecha remite a un Gabino muy joven a quien, a tenor del nombramiento posterior, podemos suponer ya vinculado (como meritorio quizá) al Departamento de Grabado, lo cual conllevaba para los alumnos más descollantes, según se lee en la Instrucción y Plan de Enseñanza, el seguimiento de estudios en la Academia ${ }^{8}$. Hay que apuntar que los contrincantes de Gabino en el concurso de 1831 eran también alumnos o ayudantes de la talla en el Departamento de Grabado, compañeros de estudios los cinco, por tanto, dentro y fuera de la Academia ${ }^{9}$.

\footnotetext{
${ }^{5}$ Núñez Vernis, Bertha, Zacarías González Velázquez (1763-1834), Madrid, 2000.

${ }^{6}$ Gabino Velázquez alcanzó los cargos de Ujier de Cámara (1834), Administrador de la Casa de la Reina (1835), de nuevo Ujier de Cámara (1842), Administrador del Patrimonio Real de Madrid (1848) y Gentilhombre de Casa y Boca (1859). En el libro de Bertha Núñez se hallarán datos acerca de sus dos matrimonios y su descendencia, y otros varios, así como referencia a obras suyas conservadas (un dibujo de «academia» del Museo de la Fábrica Nacional de Moneda y Timbre, 1830; vaciados en yeso y un modelo en cera), todas de sus años de estudiante. Según Núñez, sus estudios en la Academia se extendieron desde 1823 hasta precisamente 1834 («entre 1823 y 1828 figuraba como asistente a la Sala del Yeso, y entre 1827 y 1834 a la del Natural»). Nada suyo hay entre los dibujos de la Academia, a juzgar por los inventarios publicados: Azcárate Luxán, Isabel, «Inventario de dibujos correspondientes a las pruebas de examen de la Real Academia de San Fernando realizadas entre los años 1818 y 1857», Academia, Madrid, LX, 1985, pp. 137-262; Azcárate Luxán, Isabel; Durá Ojea, $\mathrm{M}^{\mathrm{a}}$ Victoria, y Rivera Navarro, Elena, «Inventario de dibujos correspondientes a pruebas de examen, premios y estudios de la Real Academia de San Fernando», Academia, Madrid, LXVI, 1988, pp. 363-478. Sólo en parte puede explicar este hecho la disposición $8^{\mathrm{a}}$ de la Instrucción y Plan de enseñanza del Departamento de Grabado, reproducida en la nota 8.

${ }^{7}$ Pedro González de Sepúlveda a su hijo Mariano, cit. por Bédat, Claude, «El grabador Mariano González de Sepúlveda. Sus progresos en el grabado y la estereotipia durante su estancia en París (1797-1803)», Numisma, Madrid, XX, 1970, p. 211.

${ }^{8}$ «7. ${ }^{\circ}$ Todos los discípulos asistirán diariamente sin excepción a la escuela del departamento todas las horas acostumbradas, trabajando en lo que se les ordene, y presentándolo a su corrección con docilidad, obediencia y exactitud; y quando los Gravadores Generales lo juzguen a propósito, con arreglo a los trabajos particulares del departamento, concurrirán a la Academia de San Fernando para su mayor progreso, particularmente aquellos que se hallan bastante adelantados para seguir el estudio del modelo vivo, por ser una de las cosas que no es fácil lograr con tanta comodidad fuera de otra Academia $[s i c] .8^{\circ}$. Después de las tareas diarias en que se ocupen los discípulos, presentarán al fin de cada año alguna muestra que acredite el estado de su adelantamiento, ya sea en la parte teórica ó en la Práctica ya en la Artística o Mecánica, la que dirigirán los Gravadores Generales a S. M. por mano del Señor Superintendente General de Real Hacienda, con su informe en que exprese las circunstancias y mérito de cada uno por donde se pueda formar una idea de su aplicación; y así mismo harán los que estén en disposición, sus oposiciones a los premios Generales de la Academia, sirviéndoles aquella obra de equivalente a la que deban presentar aquel año a S. M.»; de la misma Instrucción citada anteriormente (Durán González, op. cit., p. 191).

Según dejó escrito Esteban Lozano, «en noviembre de 1831 fue cuando se estableció definitivamente una clase de grabado de medallas en la Academia de San Fernando, clase que desde la muerte de Prieto no existía, estando reducida la enseñanza del arte al Departamento de la Moneda, donde adquirían los alumnos asistentes a él más bien los conocimientos prácticos o mecánicos que los teóricos y peculiares del arte»; véase Portela Sandoval, Francisco José, «En torno al arte de la medalla o del grabado en hueco en España: dos informes de José Esteban Lozano», Anales de Historia del Arte, Madrid, II, 1990, p. 189.

${ }^{9}$ Barrio (m. en 1855) fue nombrado por R. O. de 25 de abril 1828 segundo alumno de primera clase, ayudante de la sección de talla, pasando en 10 de octubre a primero. Plañiol (m. en 1842), por R. O. de 25 de abril de 1828 , tercer alumno de primera clase, ayudante de la sección de talla, ascendiendo en 10 de octubre de ese año a segundo alumno. González
}

$A E A$, LXXVII, 2004, 307, pp. 301 a 326 

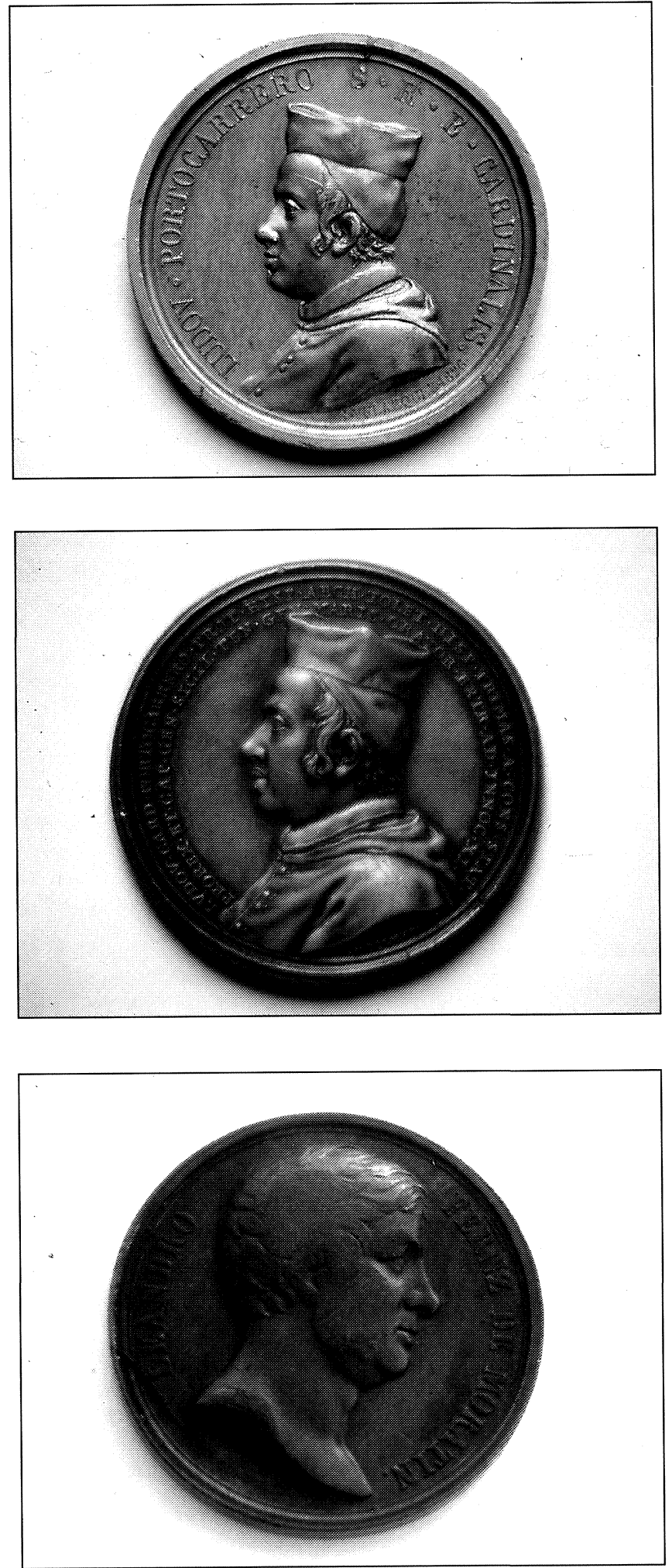

Fig. 1. G. Velázquez: medalla del cardenal Portocarrero (Colección particular, Madrid).

Fig. 2. G. Hamerani: medalla del cardenal Portocarrero, anverso (Colección particular, Madrid).

Fig. 3. B. Coromina: medalla de L. F. de Moratín (prueba de anverso; colección particular, Madrid).

AEA, LXXVII, 2004, 307, pp. 301 a 326 
Con tales estudios, por lo demás, parece vinculada esta acuñación que ahora deja de ser inédita: se trata de una réplica del anverso de la famosa medalla de Giovanni Hamerani dedicada en 1678 al cardenal Luis Portocarrero, sin duda una de las más notables creaciones de la medallística italiana de su siglo en relación con un personaje español (Fig. 2). Desde luego, las variaciones introducidas por Velázquez son sustanciales: aparte de la supresión de pequeños detalles (rizos sobre la frente, adorno del solideo), el cardenal aparece notablemente rejuvenecido, sin bigote (aunque sí con la mosca) y con un leve aire porcino en la blandura de sus rasgos. Además, al cambiar el formato de la leyenda, que queda reducida al mero enunciado del nombre y jerarquía eclesiástica principal (ajustándose así, por cierto, mucho mejor al diseño habitual de medallas de Giovanni Hamerani), la figura deja de superponerse a ella sin que varíen las relaciones de espacio. Con lo cual queda libre una mayor extensión del campo, menoscabando el dominio del busto sobre el conjunto en comparación con el original.

Nos hallamos, en suma, ante un ejercicio de grabado que compagina la intención conmemorativa de una celebridad histórica ${ }^{10}$ con el estudio de una medalla justamente admirada, obra de uno de los artistas insignes erigidos en modelo para la enseñanza tanto en la Academia como en el Departamento de Grabado ${ }^{11}$. En tal sentido, es interesante que parte de la actividad didáctica de este Departamento se orientase a la conmemoración de personalidades consideradas ilustres ${ }^{12}$. Inserta en ese contexto, el ensayo de Gabino Velázquez puede conectarse con las muestras de interés de los organismos oficiales y particulares españoles por la formación de una galería o «historia metálica» nacional, a imitación de las varias y bien nutridas emitidas en Francia poco antes (que en ocasiones incluyen personajes españoles). La generalización del interés por este tipo de conmemoraciones era por entonces creciente en sectores instruidos, en cuyo seno, sobre todo en ambientes liberales, se echaba en falta la existencia de tal colección como desdoro patrio ${ }^{13}$.

de Miranda, por R. O. de 2 de junio de 1828, tercer ayudante de segunda clase. Coromina (1808-1867), por R. O. De 16 de octubre de 1828, tercer alumno de segunda clase, sección de talla, y en 23 de marzo de 1830, segundo alumno; datos proporcionados por Herrera, op. cit., pp. 467, 491, 478 y 472 respectivamente.

${ }^{10}$ Acerca del famoso prelado, nacido en Palma del Río (Córdoba) en 1629 y fallecido en Madrid en 1709 tras una brillantísima carrera política que le encumbró a las más altas instancias del gobierno de la Monarquía hispana, no es necesario extenderse aquí. Algunos datos sobre su biografía y la medalla de Hamerani reunió en un artículo Pardo Canalís, Enrique, «El cardenal Portocarrero y la medalla de Juan Hamerano», Goya, Madrid, n. ${ }^{143,}$ 1978, pp. 278-79. Para completar provisionalmente la iconografia medallística del cardenal hay que mencionar una pequeña pieza de celebración, con retrato más bien imaginario en el anverso y en le reverso la inscripción: JE SVIS/ PLVS GRAND/ QUE/ RICHELIEV/ ET/ MAZARIN/ MDCC/ M.OCTO·. Alusiva sin duda a su intervención decisiva a favor de las aspiraciones al trono español de quien llegaría a ser Felipe V, la reproduce Van Loon, Gerard, Histoire métallique des XVII provinces des Pays-Bas, La Haya, 1732-37, vol. IV, pp. 310-11; también, en impronta, Herrera, Adolfo, Medallas españolas («medallas personales», vol. II, Madrid, 1901).

${ }^{11}$ Se da la circunstancia curiosa de que al menos dos ejemplares de esta medalla, junto a otras muchas del mismo autor, fueron adquiridas en nombre de Carlos III de la colección de Tomás Francisco Prieto, con el designio de que «pudieran servir de modelo a los alumnos pensionados de la escuela de grabado fundada por el mismo Prieto en el año 1772»; Bédat, Claude, «El grabador general Tomás Francisco Prieto (1716-1782)», Numisma, Madrid, XIX, 1969, p. 108. Estas adquisiciones pasaron posteriormente, parece, al Departamento de Grabado, subsistiendo en parte todavía en la Fábrica Nacional de Moneda y Timbre.

${ }^{12}$ Un Inventario general del Departamento de Grabado y construcción de instrumentos y máquinas para la moneda (transcrito también en Durán González, op. cit., pp. 164-67), fechado en 1826, incluye en el apartado de modelos «once cabezas de hombres célebres, modeladas por los discípulos del Departamento, puestas en marcos de cristal», «diez pruebas en yeso, sacadas de los troqueles grabados por los referidos discípulos», así como otros hechos en cera. Pero no especifica que los retratados fueran españoles.

${ }^{13}$ De ese interés generalizado es prueba la buena aceptación que tuvo más tarde, a decir de sus impulsores, la iniciativa de la Sociedad Numismática Matritense de suplir con medios privados lo que no cumplían los públicos, procediendo a la emisión de la serie. El autor de esta nota lo es también de un estudio de esta empresa y algunas de sus implicaciones («Las medallas de la Sociedad Numismática Matritense», Boletín del Museo Arqueológico Nacional, Madrid, XVII, 1999, pp. 279-93). En él sugiero que medallas como la de Bartolomé Coromina dedicada a Leandro Fernández de Moratín (fig. 3; $42 \mathrm{~cm}$. de diámetro; el reverso lo ocupa la inscripción NACIO EN MADRID/ EN 1760/ FALLECIO EN PARIS/ EN 1828/ DEPARTAMENTO DE GRABADO/ 1841) constituyen la materialización de los esfuerzos orientados en esa direc-

AEA, LXXVII, 2004, 307, pp. 301 a 326 
Habida cuenta de que ésta que doy a conocer es de momento la única muestra de su trabajo, y más aún cuando no alcanzó realización efectiva (hasta donde podemos saber; de hecho, ni siquiera podemos estar seguros de que se proyectó que la tuviera), está claro que no será con ella que Gabino González Velázquez dejará de ser poco más que un nombre en la historia del grabado en hueco en España. Pero es, repito, precisamente por ese nombre suyo, tanto como por la posibilidad de darle cuerpo histórico y situarlo con alguna claridad en el horizonte de los grabadores de su tiempo, por lo que conviene reunir cuanto sea posible acerca de su persona. Hay que insistir en la importancia de perfilar la biografía y labor de estos artistas, también de las figuras secundarias, si alguna vez va a ser posible confeccionar un relato histórico, sobre cimientos sólidos, de la medalla española del siglo XIX.

José Luis ARIAS Bonel

\section{CAPUT SANCTI IHOHANIS BAPTISTE IN DISCO EN UN ALABASTRO GÓTICO INGLÉS DE LA ANTIGUA COLECCIÓN MASSÓ}

Entre los fondos museográficos de la antigua colección particular de la familia Massó, conocida sobre todo por su colección etnográfica, sorprende la presencia de una interesante placa gótica de alabastro inglés de pequeñas dimensiones que, sin tratarse de una pieza de extraordinaria calidad, por su iconografía es obra de gran interés ${ }^{1}$.

La pieza responde al tipo conocido con el nombre de Cabezas de San Juan ${ }^{2}$. Concebida como obra única y no como parte constituyente de un retablo, está tallada en un altorrelieve que muestra la cabeza de San Juan Bautista dispuesta sobre una bandeja circular, debajo de la cual, con unas dimensiones menores, figura la imagen de Cristo resucitado saliendo del sepulcro, con su mano derecha colocada sobre la herida abierta en el costado, y la izquierda, mutilada, en actitud de intentar levantarla ${ }^{3}$ (Fig. 1).

El relieve, que llegó hasta nuestros días bastante fragmentado, denuncia su factura inglesa. En efecto, estilísticamente hablando, la talla constituye un reflejo desvirtuado de los modelos de Cabezas de San Juan Bautista que habían introducido los talleres ingleses a comienzos del siglo $\mathrm{xv}$, modelos que se distinguían por su alta calidad, ostensible en una cierta exquisitez y minuciosidad en el tratamiento de las formas y en la elaboración de los detalles. A partir de la segunda mitad del siglo xv la producción de alabastros entra en un proceso de

ción, aun en momentos en que la medallística oficial quedaba en gran parte en manos francesas. No eran pocas las dificultades técnicas y económicas en España para el grabado de medalla en la época de Gabino Velázquez. Imposible competir en técnica, maquinaria y riqueza de diseño con Francia, hacia donde se dirigió la iniciativa española. Mejor producto y más barato, como aún reconocía, mucho tiempo después y con cierta amargura, un erudito entusiasta (Castellanos, Basilio Sebastián, El progreso en las Bellas Artes. Estudio de esculto-grabado y grabado comercial e industrial fundado por D. Victorino González y Fernández, Madrid, 1880, pp. 17-18).

\footnotetext{
${ }^{1}$ Esta pieza se conserva actualmente en el recién creado Museo Massó (Bueu, Pontevedra), en donde ingresó fruto de la compra que la Xunta de Galicia realizó en el año 1995 de la antigua colección de la familia Massó.

${ }^{2}$ Sobre estas piezas, denominadas Cabezas de San Juan véase Hope, W.H.: «On the sculptured alabaster tablets called Saint John's Heads», Archeologia, LII, 1890; Cheetham, F.W.: Medieval English alabaster carvings in the Castle Museum Nottingham, Nottingham: Ed. Derry and sons limited, 1962, p. 49-50; idem, 1984, pp. 317-332; Idem, English Medieval Alabasters. With a Catalogue of Collection in the Victoria and Albert Museum, Oxford: Ed. Phaidon Press Limited, 1984, pp. 317-332.

${ }^{3}$ Sus medidas son: alto: $27^{\prime} 5 \mathrm{~cm}$, largo: $14 \mathrm{~cm}$, y de ancho: $1 \mathrm{~cm}$. Su estado de conservación es bastante malo, ya que se encuentra fragmentada por la mitad. Está colocada sobre una peana de madera y sujeta a ambos lados por grapas metálicas.
}

AEA, LXXVII, 2004, 307, pp. 301 a 326 